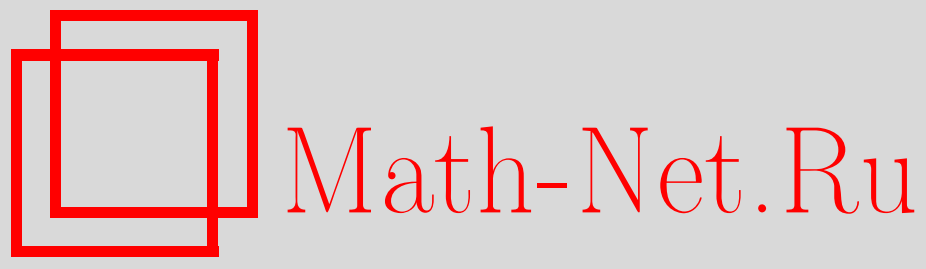

Н. Б. Енгибарян, Асимптотические и структурные теоремы для уравнения марковского восстановления, Теория вероятн. и ее примен., 2003, том 48, выпуск 1, 62-77

DOI: https://doi.org/10.4213/tvp301

Использование Общероссийского математического портала MathNet.Ru подразумевает, что вы прочитали и согласны с пользовательским соглашением

http://www . mathnet.ru/rus/agreement

Параметры загрузки:

IP: 52.6 .47 .48

26 апреля 2023 г., 13:50:02

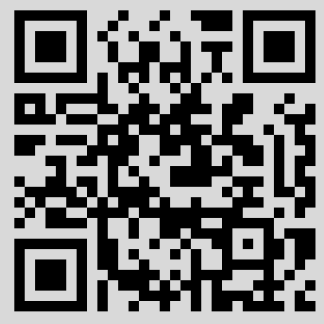


(C) $2003 \mathrm{r}$.

ЕНГИБАРЯН Н. Б.*

\section{АСИМПТОТИЧЕСКИЕ И СТРУКТУРНЫЕ ТЕОРЕМЫ ДЛЯ УРАВНЕНИЯ МАРКОВСКОГО ВОССТАНОВЛЕНИЯ}

Рассматривается уравнение многомерного восстановления

$$
\varphi(t)=g(t)+\int_{0}^{t}[d F(x)] \varphi(t-x) .
$$

Здесь $g \in L_{1}^{n}(0 ; \infty), F(t)=\left(F_{i j}(t)\right)_{i, j=1}^{n}(n<\infty), F(t)=0$ при $t \leqslant 0$, $F(t) \uparrow, r(A)=1$, где $A=F(+\infty), r(A)$ - спектральный радиус матрицы $A$. В частном случае уравнения марковского восстановления имеем $\sum_{i=1}^{n} F_{i j}(+\infty)=1$.

Предполагается, что матрица $A$ неразложима и некоторая сверточная степень меры $d F$ обладает нетривиальной абсолютно непрерывной компонентой. Доказано, что тогда решение уравнения марковского восстановления имеет вид: $\varphi(t)=\mu+\rho(t)+\psi(t)$, $\rho \in C_{0}^{n}[0 ; \infty), \psi \in L_{1}^{n}(0 ; \infty)$. Если мера $d F$ обладает конечным вторым моментом, то $\rho \in L_{1}^{n}(0 ; \infty)$. Получены явные формулы для $\mu$ и $\sigma=\int_{0}^{\infty}[\varphi(t)-\mu] d t$. Отсюда, в частности, следует асимптотическая формула для $\int_{0}^{t} \varphi(x) d x$.

Ключевые слова и фразы: структура и асимптотика решения уравнения многомерного восстановления.

\section{$\S 1$. Введение}

Рассмотрим уравнение марковского восстановления (УМВ), связанное с полумарковским процессом $\xi(t)$ (см. [1]-[6]):

$$
\varphi(t)=g(t)+\int_{0}^{t}[d F(x)] \varphi(t-x)
$$

Здесь $g \in L_{1}^{n}(0 ; \infty), F(t)=\left(F_{i j}(t)\right)_{i, j=1}^{n}(n<\infty)$ - полумарковская матрица:

$$
F(t)=0, \quad t \leqslant 0 ; \quad F(t) \uparrow ; \quad \sum_{i=1}^{n} F_{i j}(+\infty)=1
$$

*Бюраканская астрофизическая обсерватория НАН РА, 378433 Бюракан, Армения; e-mail: yengib@bao.sci.am 
Матрицу-функцию $F$ мы назовем предъядром уравнения (1.1).

В скалярном случае $(n=1) F$ является распределением вероятностей неотрицательной случайной величины.

Функция марковского восстановления $H$ определяется из уравнения

$$
H(t)=F(t)+\int_{0}^{t}[d F(x)] H(t-x) .
$$

Теория скалярного уравнения (1.1) (и (1.3)) достаточно хорошо развита в рамках теории восстановления (см. [7]-[9]). К центральным результатам асимптотической теории скалярного уравнения восстановления (УВ) относятся основная теорема восстановления Д.Блекуэлла и асимптотическая теорема В. Смита. Из теоремы Блекуэлла следует существование предела $\varphi(+\infty)$ в случае, когда распределение $F$ неарифметическое, а функция $g$ непосредственно интегрируема по Риману.

В теореме Смита построены первые два члена асимптотики функции восстановления в случае $\nu_{2}<\infty$. Здесь и в дальнейшем используется следующее обозначение для моментов меры $d F$ :

$$
\nu_{k}=m_{k}(d F)=\int_{0}^{\infty} t^{k} d F(t) \leqslant \infty, \quad k \geqslant 0 .
$$

Теорема 1.1 (В. Смит). Пусть $F$ - неарифметическое распределение и $\nu_{1}<\infty$. Тогда

$$
H(t)-\alpha t \rightarrow \beta, \quad t \rightarrow \infty,
$$

где $\alpha=\nu_{1}^{-1}, \beta=\nu_{2} /\left(2 \nu_{1}^{2}\right)-1 \leqslant \infty$.

Eсли $\nu_{2}<\infty$, mo

$$
H(t)=\alpha t+\beta+o(1), \quad t \rightarrow \infty .
$$

Напомним, что распределение $F$ называется неарифметическим, если оно не сосредоточено на некоторой решетке $\{k r\}, r>0, k=0,1, \ldots$.

Широкий и важный класс вероятностных распределений составляют распределения $F$ абсолютно непрерывного типа, некоторая сверточная степень $F^{p *}$ которых содержит нетривиальную абсолютно непрерывную компоненту.

В работе автора [10] доказана структурная теорема для скалярного уравнения восстановления. Из этой теоремы следует, что если $F$ распределение абсолютно непрерывного типа, то предел $\varphi(\infty)$ существует при значительно более слабом ограничении на $g$, чем условие непосредственной интегрируемости по Риману, а именно: $g \in L_{1}(0 ; \infty)$, $g \in M(0 ; \infty), g(+\infty)=0$.

Вопросы асимптотического поведения решения системы (1.1) в $+\infty$ изучены в ряде работ (см. [1]-[6]). Они представляют известный интерес 
в теории надежности, теории массового обслуживания, теории стохастических автоматов, исследовании операций, теории ветвящихся процессов и др. Одним из основных фактов в этом направлении является следующее обобщение на многомерный случай теоремы Блекуэлла, которое в той или иной степени общности было доказано в работах [2]-[5], [11] и др.

Теорема 1.2. Пусть $\xi(t)$ - неприводимый возвратный полумарковский прочесс, $n \leqslant \infty$. Тогда

$$
H_{i j}(t)-H_{i j}(t-r) \longrightarrow \frac{r}{\mu_{j j}} \quad n p u \quad t \rightarrow \infty, \quad \forall i, j, \forall r>0
$$

где $\mu_{i i}$ - среднее время возврашения в $i$-е состояние.

Получить асимптотические теоремы для УМВ (1.3) не удавалось и пришлось ограничиться асимптотическими формулами для преобразования Лапласа-Стилтьеса функции $H^{\prime}$ (см. [1]).

В работе автора [6] предложен метод треугольной факторизации матричной структуры конечномерного УМВ (1.1). Этот подход дает возможность распространить, в достаточно общей форме, на многомерный случай различные свойства решения скалярного УВ.

Аналогично элементарной теореме восстановления, теорема Смита не приводит к адекватному описанию свойств решения УВ (1.1), если свободный член является (в том или ином смысле) интегрируемой функцией на полуоси. Поэтому представляют интерес такие формы асимптотических и предельных теорем, которые допускают распространение на обшие уравнения (1.1) с интегрируемым свободным членом.

В настоящей работе путем применения подходов и результатов работ [6] и [10] получены новые асимптотические и структурные теоремы по скалярному и многомерному УВ (1.1).

\section{§ 2. Обозначения и вспомогательные предложения}

2.1. Алгебраические объекты. Пусть $\mathbf{R}^{n}$ и $\mathbf{R}_{n}$ - пространства $n$-мерных вектор-столбцов и вектор-строк соответственно; $\mathbf{R}^{n \times n}-$ алгебра $(n \times n)$-матриц с единицей $I$. Через $A^{\mathrm{T}}$ обозначается матрица или вектор, транспонированные к $A$. Неравенства типа $>$ и $\geqslant$ между матрицами или векторами понимаются поэлементно. Запись $A \succ B$ означает, что $A \geqslant B, A \neq B$.

Через $r(A)$ обозначается спектральный радиус матрицы $A$.

Введем в рассмотрение класс $P_{\mathrm{N}} \subset \mathbf{R}^{n \times n}$ неотрицательных неразложимых (неприводимых) матриц (см. [12]). Согласно теореме ПерронаФробениуса, число $\lambda=r(A)$ является простым собственным значением матрицы $A \in P_{\mathrm{N}}$, которому соответствуют положительные, правый и 
левый, собственные векторы:

$$
A \eta=\lambda \eta, \quad \varsigma A=\lambda \varsigma, \quad \eta \in \mathbf{R}^{n}, \quad \varsigma \in \mathbf{R}_{n}, \quad \eta, \varsigma>0 .
$$

2.2. Классы функций. Введем в рассмотрение следующие банаховы пространства определенных на $[0 ; \infty)$ скалярных функций: $L_{1}, M, M_{0}, C_{0}$, VAR. Здесь $M$ - пространство ограниченных в существенном функций; $M_{0} \subset M$ и $C_{0} \subset C[0 ; \infty)$ состоят из функций $f$ таких, что $f(\infty)=0$. Пространство VAR $\subset M$ состоит из непрерывных слева функций $f$ ограниченной вариации, для которых $f(0)=0$, с нормой $\|f\|_{\text {VAR }}=\mathrm{V}_{0}^{\infty} f$.

Пусть $E$ - одно из указанных банаховых пространств.

Через $E^{n}$ и $E^{n \times n}$ обозначаются пространства вектор-столбцов и матриц-функций соответственно с элементами из $E$.

Обозначим через MON $\subset$ VAR конус неубывающих функций.

Нами будут рассмотрены также линейные топологические пространства $E_{\text {loc }}$ определенных на $[0 ; \infty)$ функций, принадлежащих $E[0 ; r]$ $\forall r<\infty$. Здесь $E[0 ; r]$ одно из пространств $C[0 ; r], L_{1}(0 ; r), M(0 ; r)$, $\operatorname{VAR}[0 ; r]$.

2.3. Свертка и моменты функций и мер. Определим свертку $f \bullet g$ :

$$
(f \bullet g)=\int_{0}^{t} f(t-x) g(x) d x
$$

где $f \in L_{\text {loc }}^{n \times n}$, а $g$ - матрица-функция или вектор-столбец с элементами из $L_{\text {loc }}$.

Введем также следующую свертку:

$$
(F * \varphi)(t)=\int_{0}^{t}[d F(x)] \varphi(t-x),
$$

где $F \in \operatorname{VAR}^{n \times n}(n \geqslant 1)$, a $g$ - матрица-функция или вектор-столен с элементами из $E$.

Имеет место следующая формула: если $F, \varphi \in \mathrm{VAR}^{n \times n}$, то

$$
(F * \varphi)(+\infty)=F(+\infty) \varphi(+\infty) \text {. }
$$

Класс $\mathrm{VAR}^{n \times n}$ представляет собой сверточную алгебру с умножением *. Введем моменты скалярных или матричных функций и мер:

$$
\tau_{k}(f)=\int_{0}^{\infty} t^{k} f(t) d t, \quad m_{k}(d F)=\int_{0}^{\infty} t^{k} d F(t), \quad k \geqslant 0 .
$$

В случае, когда некоторые компоненты $f$ знакопеременные, интегралы в выражении для $\tau_{k}(f)$ предполагаются абсолютно сходяшимися. Если $f \geqslant 0$, то ее моменты (покомпонентно) могут принимать также бесконечное значение. 
Если $\tau_{k}(|f|)<\infty, \tau_{k}(|g|)<\infty$, то $\tau_{k}(|f \bullet g|)<\infty$ и

$$
\tau_{k}(f \bullet g)=\sum_{p=0}^{k} C_{k}^{p} \tau_{p}(f) \tau_{k-p}(g) .
$$

2.4. Классы операторов. Обозначим через $J$ единичный оператор. Введем следующие классы $\Omega$ и $\Omega_{\text {a }}$ интегральных операторов свертки. Если $\breve{F} \in \Omega$, то $\breve{F} \varphi=F * \varphi, F \in \mathrm{VAR}^{n \times n}$.

Класс $\Omega_{\mathrm{a}} \subset \Omega$ состоит из операторов с абсолютно непрерывными предъядрами: $\breve{U} \varphi=U \bullet \varphi, U \in L_{1}^{n \times n}$.

Операторы из указанных классов ограниченно действуют в пространствах $E$ и в ряде других пространств.

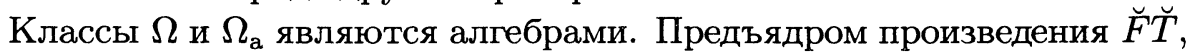
где $\breve{F}, \breve{T} \in \Omega$, является свертка $F * T$ их предъядер. Ядром произведения $\breve{U} \breve{V}$, где $\breve{U}, \breve{V} \in \Omega_{\text {a }}$, является свертка $U \bullet V$ их ядер.

Пусть $\breve{F} \in \Omega$, где $F \in \mathrm{MON}^{n \times n}$. Пусть $A=F(+\infty), \lambda=r(A)$. Если $\lambda<1$, то оператор $J-\breve{F}$ обратим в пространствах $E$; если $\lambda=1$, то $J-\breve{F}$ необратим.

2.5. Существование моментов. Рассмотрим вопрос существования моментов решения уравнения вида (1.1) с обратимым оператором, в зависимости от конечности моментов у $d F$ и $g$.

Перепишем уравнение (1.1) в операторной форме

$$
(J-\breve{F}) \varphi=g .
$$

Рассмотрим также тот частный случай уравнения (2.3), когда предъядро $F$ абсолютно непрерывно, $F^{\prime}(x)=U(x) \in L_{1}^{n \times n}$ :

$$
\varphi(t)=\gamma(t)+\int_{0}^{t} U(t-x) \varphi(t) d t, \quad \text { или } \quad(J-\breve{U}) \varphi=\gamma .
$$

Лемма 2.1. а) Пусть в уравнении (2.4) (ядро которого может быль знакопеременным) оператор $J-\breve{U}$ обратим в $L_{1}^{n} u \tau_{k}(|U|)<\infty$, $\tau_{k}(|\gamma|)<\infty$ при некотором $k \geqslant 0$. Тогда $\tau_{k}(|\varphi|)<\infty$.

б) Пусть в уравнении (1.1) $F \in \mathrm{MON}^{n \times n}, \quad r[F(+\infty)]<1$, $m_{k}(d F)<\infty, \tau_{k}(|g|)<\infty, k \geqslant 0$. Тогда $\tau_{k}(|\varphi|)<\infty$.

Д о к а з а т л ь с т в о. Лемма будет доказана методом (неполной) индукции. Конечность нулевого момента решения (2.4) следует из обратимости оператора $J-\breve{U}$ в $L_{1}^{n}$.

Предположим, что $\varphi$ обладает конечными моментами вплоть до порядка $p-1,1 \leqslant p \leqslant k$. Тогда $U_{q}(t) \equiv t^{q} U(t) d t \in L_{1}^{n}, \gamma_{q}(t) \equiv t^{q} \gamma(t) \in L_{1}^{n}$, $q \leqslant k ; \varphi_{q}(t) \equiv t^{q} \varphi(t) \in L_{1}^{n}, q<p$. Умножая обе части (2.4) на $x^{p}$, получаем соотношение

$$
\varphi_{p}=\left[\gamma_{p}+\sum_{q=0}^{p-1} C_{p}^{q} U_{p-q} * \varphi_{q}\right]+U * \varphi_{p}
$$


которое представляет собой уравнение относительно $\varphi_{p}$ с суммируемым свободным членом и обратимым оператором. Поэтому $\varphi_{p} \in L_{1}^{n}$. Второе утверждение леммы доказывается сходным образом. Лемма доказана.

\section{§3. Скалярное уравнение}

3.1. Преобразование уравнения с абсолютно непрерывным предъядром. Рассмотрим скалярное УВ (вида (2.4))

$$
\varphi=\gamma+u \bullet \varphi
$$

в случае, когда ядро $u$ является плотностью распределения неотрицательной случайной величины:

$$
u \in L_{1}^{+}, \quad u \geqslant 0, \quad \int_{0}^{\infty} u(t) d t=1 .
$$

Уравнение (3.1) с условиями (3.2) является частным случаем скалярного уравнения (1.1), когда предъядро $F$ абсолютно непрерывно. Тогда $F^{\prime}(t)=u(t), H^{\prime}(t)=h(t)$. Плотность восстановления $h$ определяется из $\mathrm{YB}$

$$
h=u+u \bullet h \text {. }
$$

Имеет место формула

$$
\varphi=\gamma+h \bullet \gamma
$$

Рассмотрим вопрос о структуре решения уравнения (3.4) при предположении конечности первого момента ядра:

$$
\tau_{1}(u)<\infty
$$

Введем функцию

$$
h_{1}(t)=h(t)-\mu
$$

где $\mu=\left[\tau_{1}(u)\right]^{-1}>0$.

Из (3.6) и (3.4) получаем уравнение:

$$
h_{1}=u_{1}+u \bullet h_{1},
$$

где $u_{1}(t)=u(t)-\mu \int_{t}^{\infty} u(x) d x$. Из (3.5) следует $u_{1} \in L_{1}$. Если $\tau_{k}(u)<\infty$, то $\tau_{k-1}\left(\left|u_{1}\right|\right)<\infty, k \geqslant 1$. Легко проверить, что

$$
\int_{0}^{\infty} u_{1}(t) d t=0
$$


Из (3.7) получаем $h_{1}=u_{1}+h \bullet u_{1}$, или

$$
h_{1}=u_{2}+u_{1} \bullet h_{1}
$$

где $u_{2}(t)=u_{1}(t)-\mu \int_{t}^{\infty} u_{1}(x) d x$.

Имеем:

$$
\begin{aligned}
& \tau_{1}(u)<\infty \Longrightarrow u_{2} \in L_{1}+C_{0} ; \quad \tau_{2}(u)<\infty \Longrightarrow u_{2} \in L_{1} ; \\
& \tau_{k}(u)<\infty \Longrightarrow \tau_{k-2}\left(\left|u_{2}\right|\right)<\infty, \quad k \geqslant 2 .
\end{aligned}
$$

Соотношение (3.9) можно рассматривать как УВ с ядром $u_{1}$. Перепишем его в операторном виде

$$
\left(J-\breve{u}_{1}\right) h_{1}=u_{2}
$$

где $\breve{u}_{1} \in \Omega_{\mathrm{a}}-$ скалярный оператор свертки с ядром $u_{1}$.

3.2. Обратимость оператора $J-\breve{u}_{1}$. В настояшем пункте будет доказана обратимость $J-\breve{u}_{1}$ в пространствах $E$. Тем самым будет установлено, что $h_{1} \in L_{1}^{+}$при $\tau_{2}(u)<\infty$.

Лемма 3.1. Oператор $J-\breve{u}_{1}$ обратим в пространствах $E$.

Д о к а з а т ел ь с т в о. Оператор $J-\breve{u}_{1}$ можно рассматривать как частный случай интегрального оператора Винера-Хопфа второго рода. Поэтому для его обратимости необходимо и достаточно, чтобы его символ удовлетворял следующим условиям (см. [13], [14]):

$$
\begin{gathered}
1-\widehat{u}_{1}(s) \neq 0, \quad-\infty<s<\infty, \\
\varkappa=\operatorname{ind}\left[1-\widehat{u}_{1}\right]=\frac{1}{2 \pi} \int_{-\infty}^{\infty} d \arg \left[1-\widehat{u}_{1}(s)\right]=0,
\end{gathered}
$$

где $\widehat{u}_{1}(s)=\int_{0}^{\infty} u_{1}(t) \exp (i s t) d t$ (преобразование Фурье на полуоси). Проверим первое из условий (3.12). Нетрудно получить формулы

$$
\begin{aligned}
1-\widehat{u}_{1}(s) & =[1-\widehat{u}(s)]\left(1+i \frac{\mu}{s}\right), \quad s \neq 0, \quad 1-\widehat{u}_{1}(0)=1 \\
1-\widehat{u}(s) & =2 \int_{0}^{\infty} u(t) \sin ^{2} \frac{s t}{2} d t-i \int_{0}^{\infty} u(t) \sin s t d t
\end{aligned}
$$

Из (3.13) следует, что если $s \neq 0$, то $\operatorname{Re}[1-\widehat{u}(s)]>0$. Поэтому $s=0$ является единственным нулем $\operatorname{Re}[1-\widehat{u}(s)]$ на вещественной оси. Первое из соотношений (3.13) показывает, что первое из условий (3.12) выполняется.

Рассмотрим теперь функцию $z=1-\widehat{u}_{1}(s) \equiv \Lambda_{1}(s)+i \Lambda_{2}(s)$, $-\infty \leqslant s \leqslant+\infty$, график которой является замкнутой параметризованной 
кривой Г в комплексной плоскости. Имеем:

$$
\begin{aligned}
& \Lambda_{1}(s)=2 \int_{0}^{\infty} u(t) \sin ^{2} \frac{s t}{2} d t+\frac{\mu}{s} \int_{0}^{\infty} u(t) \sin s t d t \\
& \Lambda_{2}(s)=\frac{2 \mu}{s} \int_{0}^{\infty} u(t) \sin ^{2} \frac{s t}{2} d t-\int_{0}^{\infty} u(t) \sin s t d t
\end{aligned}
$$

Пусть $\Lambda_{2}\left(s_{0}\right)=0, s_{0} \neq 0$. Тогда

$$
\Lambda_{1}\left(s_{0}\right)=2\left(1+\frac{\mu^{2}}{s_{0}^{2}}\right) \int_{0}^{\infty} u(t) \sin ^{2} \frac{s_{0} t}{2} d t>0
$$

С учетом (3.13) заключаем, что кривая $\Gamma$ расположена в комплексной плоскости с разрезом вдоль отрицательной полуоси $(-\infty ; 0]$. Поэтому индекс $\varkappa=0$. Лемма доказана.

Отметим, что обратимость оператора вида $J-\breve{u}_{1}$ несколько иным способом была установлена в [15].

Рассмотрим теперь уравнение $(3.7)$ при $\tau_{2}(u)<\infty$. Из обратимости $J-\breve{u}_{1}$ и формул (3.10), (3.11), (3.6) вытекает следующая лемма.

Лемма 3.2. Пусть ядро и скалярного уравнения (3.1) удовлетворяет условиям (3.2) и обладает конечным вторым моментом. Тогда справедливы следующие утверждения.

а) Плотность восстановления $h$ имеет вид

$$
h(t)=\left[\tau_{1}(u)\right]^{-1}+h_{1}(t), \quad h_{1} \in L_{1} .
$$

Если ядро и обладает конечньми моментами до порядка $k$ включительно, то

$$
\tau_{k-2}\left(\left|u_{2}\right|\right)<\infty, \quad \tau_{k-2}\left(\left|h_{1}\right|\right)<\infty, \quad k \geqslant 2
$$

б) Если $\gamma \in L_{1}, \tau_{1}(|\gamma|)<\infty$, то решение уравнения (3.1) имеет структуру

$$
\varphi(t)=\left[\tau_{1}(u)\right]^{-1} \int_{0}^{\infty} \gamma(x) d x+\varphi_{1}(t), \quad \varphi_{1} \in L_{1} .
$$

Заметим, что из полученных выше результатов следует также теорема 1.2 в частном случае уравнения $(3.1)$ с $\tau_{1}(u)<\infty$.

3.3. Случай $F_{\mathrm{a}}(+\infty)>0$. Целью настоящего пункта является распространение леммы 3.2 на скалярное уравнение (1.1) в случае, когда предъядро $F$ является функцией распределения неотрицательной случайной величины и обладает нетривиальной абсолютно непрерывной компонентой. Тогда

$$
F=F_{\mathrm{a}}+F_{1} \in \mathrm{MON}, \quad \lambda \equiv F(+\infty)=1, \quad F_{\mathrm{a}}(+\infty)>0 .
$$


Здесь $F_{\text {a }}$ - абсолютно непрерывная компонента $F$, а $F_{1}$ - сумма сингулярной и дискретной компонент.

Мы будем следовать подходу работы [10]. Перепишем скалярное уравнение $(1.1)$ в операторном виде $(2.3)$. В силу $F_{1}(+\infty)<1$ оператор $J-\breve{F}_{1}$ обратим в $E$ и (2.3) сводится к уравнению $(3.1)$, где ядро $u$ и свободный член $\gamma$ определяются из следующих уравнений:

$$
\begin{aligned}
& u(t)=F_{\mathrm{a}}^{\prime}(t)+\int_{0}^{t} u(t-x) d F_{1}(x), \\
& \gamma(t)=g(t)+\int_{0}^{t} \gamma(t-x) d F_{1}(x) .
\end{aligned}
$$

Из (3.17) легко следует, что $u$ удовлетворяет условиям (3.2). Применяя к уравнениям (3.18) и (3.19) лемму 2.1, мы делаем вывод о наличии моментов соответствующих порядков у функций $u$ и $\gamma$ в зависимости от свойств $F$. Объединяя полученные результаты с теоремой 5.1 работы [10], приходим к следующей теореме.

Теорема 3.1. Пусть предъядро скалярного уравнения восстановления (1.1) (или (2.3)) обладает свойствами (3.17) и $g \in L_{1}$. Тогда решение уравнения имеет структуру

$$
\varphi(t)=\nu_{1}^{-1} \int_{0}^{\infty} g(x) d x+\rho(t)+\psi(t) \equiv \nu_{1}^{-1} \int_{0}^{\infty} g(x) d x+\omega(t),
$$

әде $\rho \in C_{0}, \psi \in L_{1} ; \nu_{k}=m_{k}(d F) \leqslant \infty, k \geqslant 1$.

Ecлu $g \in L_{1} \cap M_{0}$ u $g(+\infty)=0$, mo

$$
\psi(\infty)=0, \quad \varphi(\infty)=\nu_{1}^{-1} \int_{0}^{\infty} g(x) d x
$$

Eсли $\nu_{2}=m_{2}(d F)<\infty, m_{1}(|g|)<\infty$, mo

$$
\rho \in L_{1}, \quad \int_{0}^{\infty} \omega(t) d t=\frac{\nu_{2}}{2 \nu_{1}^{2}} \tau_{0}(g)-\nu_{1}^{-1} \tau_{1}(g) .
$$

Eсли $\nu_{k}=m_{k}(d F)<\infty, \tau_{k-1}(|g|)<\infty, k \geqslant 2$, mo $\tau_{k-2}(|\omega|)<\infty$.

\section{§4. Уравнение марковского восстановления (УМВ)}

В настоящем параграфе рассматривается система (1.1) при следующих условиях критичности на матричное предъядро $F$ :

$$
F \in \mathrm{MON}^{n \times n}, \quad A \equiv F(+\infty) \in P_{\mathrm{N}}, \quad \lambda \equiv r(A)=1 .
$$

Из (4.1) следует (см. (2.1)), что существуют положительные векторы $\eta$ и $\varsigma$ такие, что

$$
A \eta=\eta, \quad \varsigma A=\varsigma, \quad \eta \in \mathbf{R}^{n}, \quad \varsigma \in \mathbf{R}_{n}, \quad \eta, \varsigma>0 .
$$


Уравнение (1.1) с условиями (4.1) называется уравнением многомерного восстановления (см. [1]). Формально оно более общее, чем УМВ, так как матрица $A$ может не быть стохастической. Однако уравнение (1.1), (4.1) простым матричным преобразованием сводится к УМВ.

Предполагается, что матрица-функция $F$ является распределением абсолютно непрерывного типа.

Применяемый факторизационный подход подробно изложен в работе [6], поэтому мы ограничимся его схематическим описанием в последуюших двух пунктах.

4.1. Факторизация матрицы $I-A$. Введем следующие подалгебры треугольных матриц $G^{ \pm}$алгебры $\mathbf{R}^{n \times n}$ :

$$
\begin{gathered}
\text { если } \quad B=\left(b_{i j}\right) \in G^{+}, \quad C=\left(c_{i j}\right) \in G^{-}, \\
\text {то } \quad b_{i j}=0, \quad j \geqslant i ; \quad c_{i j}=0, \quad j<i .
\end{gathered}
$$

Обозначим через $P_{+}$и $P_{-}$проекторы, переводяшие $\mathbf{R}^{n \times n}$ в $G^{+}$и $G^{-}$ соответственно.

Рассмотрим факторизацию

$$
I-A=(I-B)(I-C),
$$

где матрица $A$ определяется согласно (4.1), $B$ и $C$ суть искомые матрицы вида (4.3).

В [6] доказано существование факторизации (4.4), где матрицы $B$ и $C$ обладают свойствами:

$$
\begin{gathered}
0 \leqslant C \in G^{-}, \quad C \eta=\eta, \quad c_{n n}=1, \quad c_{k k}<1, \quad k<n \\
0 \leqslant B \in G^{+}, \quad \varsigma-\varsigma B=\left(0, \ldots, 0, \varsigma_{n}\right) .
\end{gathered}
$$

Имеем: $(I-B)^{-1}=I+\widetilde{B} \equiv I+B+\cdots+B^{n-1}$.

4.2. Факторизация оператора $J-\breve{F}$. Введем следующие подалгебры $\Omega^{ \pm}$операторной алгебры $\Omega$, введенной в п. 2.4: если $\breve{V} \in \Omega^{+}$, $\breve{W} \in \Omega^{-}$, то их предъядра $V(t)=\left(v_{i j}(t)\right), W(t)=\left(w_{i j}(t)\right)$ принимают значения из $G^{+}$и $G^{-}$соответственно, т.е. $v_{i j}(t)=0, j \geqslant i ; w_{i j}(t)=0$, $j<i$.

Рассмотрим следующую факторизацию оператора $J-\breve{F}$, фигурирующего в (2.3):

$$
J-\breve{F}=(J-\breve{V})(J-\breve{W})
$$

где $\breve{V} \in \Omega^{+}, \breve{W} \in \Omega^{-}$.

Из (4.6) получаем следующую систему уравнений гауссовской факторизации относительно искомых предъядер $V, W$ :

$$
V=P_{+} F+P_{+}(V * W), \quad W=P_{-} F+P_{-}(V * W) .
$$


Рассмотрим следуюшие последовательные приближения:

$$
\begin{aligned}
V_{k+1} & =P_{+} F+P_{+}\left(V_{k} * W_{k}\right), \\
W_{k+1} & =P_{-} F+P_{-}\left(V_{k} * W_{k}\right),
\end{aligned}
$$

Пусть выполнены условия (4.1). Тогда индукцией по $k$ доказываются неравенства $0 \leqslant V_{k} \leqslant B, 0 \leqslant W_{k} \leqslant C$, где $B$ и $C$ суть матрицы, фигурирующие в факторизации (4.4). Из монотонного возрастания по конусу MON и ограниченности последовательностей $V_{k}, W_{k}$ следует их поэлементная сходимость в пространстве VAR.

Теорема 4.1. Пусть предъядро системы (2.3) удовлетворяет условиям (4.1). Тогда справедливы следуюшие утверждения:

а) имеет место бакторизачия (4.6); предъядра операторов $\breve{V} \in \Omega^{+}, \breve{W} \in \Omega^{-}$обладают свойствами:

$$
V, W \in \mathrm{MON}, \quad V(+\infty)=B, \quad W(+\infty)=C ;
$$

матриұы $B$ и $C$ обладают свойствами (4.5).

б) для произвольного әлемента $F_{i j}$ матричы $F$ существуют $\alpha, \beta \in$ MON,$\alpha(+\infty)>0$ maкue, что

$$
W_{n n}=\alpha * F_{i j}+\beta .
$$

Д о к а з а т е л ь с т в о. Нам остается доказать (4.10). Достаточно рассмотреть случай $F_{i j}(+\infty)>0$. Пусть $\widetilde{F}-$ матрица, полученная из $F$ аннулированием элемента $F_{i j}$. В силу неприводимости матрицы $A$ имеем $\widetilde{\lambda} \equiv r(\widetilde{A})<1$, где $\widetilde{A}=\widetilde{F}(+\infty) \prec A$. Пусть $\widetilde{V}_{k}, \widetilde{W}_{k}$ определяются из итераций вида (4.8), в которых матрица $F$ заменена на $\widetilde{F}$. Очевидно, $\widetilde{V}_{k} \leqslant V_{k}(\leqslant V), \widetilde{W}_{k} \leqslant W_{k}(\leqslant W)$. Поэтому итерации $\widetilde{V}_{k}, \widetilde{W}_{k}$ сходятся: $\widetilde{V}_{k} \rightarrow \widetilde{V} \leqslant V, \widetilde{W}_{k} \rightarrow \widetilde{W} \leqslant W$. Имеем $I-\widetilde{A}=(I-\widetilde{B})(I-\widetilde{C})$, где $\widetilde{B}=\widetilde{V}(+\infty), \widetilde{C}=\widetilde{W}(+\infty)$. Ясно, что $(\widetilde{C})_{n n}<1$. В противном случае обратимая матрица $I-\widetilde{A}$ имела бы необратимый сомножитель $I-\widetilde{C}$. С учетом равенства $c_{n n}=1$ заключаем, что $\left(\widetilde{W}_{k}\right)_{n n}(+\infty)<\left(\widetilde{W}_{k}\right)_{n n}(+\infty)$, начиная с некоторого $k$. Поскольку функция $\left(W_{k}\right)_{n n}$ получается из элементов матрицы $F$ путем применения операций сложения и свертывания, то $\left(W_{k}\right)_{n n}=\alpha * F_{i j}+\left(\widetilde{W}_{k}\right)_{n n}$, где $\alpha(+\infty)>0$. Ясно, что слагаемое $\alpha * F_{i j}$ сохраняется в выражении $W_{n n}$. Теорема доказана.

Из $V \in \Omega^{+}$следует, что

$$
(J-\breve{V})^{-1}=J+\breve{V}+\breve{V}^{2}+\cdots+\breve{V}^{n-1} \equiv J+\tilde{V} .
$$

Из свойств (4.5) следует, что все скалярные операторы $J-W_{k k}$ обратимы, за исключением $J-W_{n n}$. 
Рассмотрим теперь вопрос существования моментов у предъядер $V, W$. Можно показать, что

$$
\left(m_{k}(d F)<\infty\right) \Longrightarrow\left(m_{k}(d V)<\infty, m_{k}(d W)<\infty\right) ; \quad k \geqslant 0 .
$$

Доказательство основано на использовании рекуррентного характера уравнений гауссовской факторизации (4.7) относительно элементов искомых матриц: эти соотношения либо чисто рекуррентны, либо представляют собой скалярные уравнения со сжимаюшими операторами $U_{j j}$, $j<n$. В последнем случае применяется лемма 2.1 .

4.3. Применение факторизации (4.6) к уравнению (2.3). Факторизация (4.6) и формула (4.11) сводят УВ (2.3) к следующей системе с треугольным матричным ядром:

$$
(J-\breve{W}) \varphi=\widetilde{g}
$$

где

$$
\tilde{g}=(I+\tilde{V}) g
$$

Имеем: $\left(m_{k}(d F)<\infty, \tau_{k}(|g|)<\infty\right) \Longrightarrow\left(\tau_{k}(|g|)<\infty\right), k \geqslant 0$.

Пусть выполнено условие

$$
\left(F_{n n}\right)_{\mathrm{a}}(+\infty)>0
$$

Из (4.7) следует, что тогда

$$
\left(W_{n n}\right)_{\mathrm{a}}(+\infty)>0 .
$$

Рассмотрим последнее из уравнений (4.13): $\breve{W}_{n n} \varphi_{n}=\widetilde{g}_{n}$. Оно представляет собой скалярное УВ относительно $\varphi_{n}$. Из (4.5) следует, что предъядро $W_{n n}$ этого уравнения является функцией распределения неотрицательной случайной величины. Поэтому при выполнении $(4.16) W_{n n}$ удовлетворяет условиям (3.17). При $g \in L_{1}^{n}$ к рассматриваемому уравнению может быть применена теорема 4.1: функция $\varphi_{n}$ имеет структуру (3.20). Остальные уравнения системы (4.13) являются рекуррентными уравнениями относительно $\varphi_{n-1}, \ldots, \varphi_{1}$ со сжимаюшими операторами. Поэтому структура вида $(3.20)$ функции $\varphi_{n}$ переходит на функции $\varphi_{n-1}, \ldots, \varphi_{1}$. Нами доказана следуюшая лемма.

Лемма 4.1. Пусть матрица-ядро уравнения (1.1) удовлетворяет условиям (4.1) и (4.16). Тогда решение имеет вид

$$
\varphi(t)=\mu+\rho(t)+\psi(t) \equiv \mu+\omega(t),
$$

где $\rho \in C_{0}^{n}, \psi \in L_{1}^{n}$.

a) Ecлu $g \in L_{1}^{n} \cap C_{0}^{n}$, mo $\psi(\infty)=0, \varphi(\infty)=\mu$.

б) Ecли $\nu_{2}=m_{2}(d F)<\infty, m_{1}(|g|)<\infty$, mo $\rho \in L_{1}^{n}, \omega \in L_{1}^{n}$.

в) Ecлu $\nu_{k}=m_{k}(d F)<\infty, \tau_{k-1}(|g|)<\infty, k \geqslant 2$, mo $\tau_{k-2}(|\omega|)<\infty$. 
4.4. Случай предъядра абсолютно непрерывного типа. Мы воспользуемся следующим определением распределения абсолютно непрерывного типа в многомерном случае $n>1$. Матрицу-функцию $F \in \mathrm{MON}^{n \times n}$ назовем распределением абсолютно непрерывного типа, если существует $p \geqslant 1$ такое, что

$$
\left(F^{p \bullet}\right)_{\mathbf{a}}(+\infty) \succ 0
$$

Через $F^{p \bullet}$ обозначена сверточная $p$-я степень $F$.

Лемма 4.2. Пусть $F \in \mathrm{MON}^{n \times n}$ является распределением абсолютно непрерывного типа и выполняется условие $A \equiv F(+\infty) \in P_{\mathrm{N}}$. Тогда существует $q \geqslant 1$ такое, ито

$$
\left(F^{q \bullet}\right)_{\mathrm{a}}(+\infty) \succ 0, \quad F^{q \bullet}(+\infty) \in P_{\mathrm{N}}
$$

Д ок азат е льст в о. Из (4.18) и неприводимости матрицы $A$ легко следует, что $\left(F^{p \bullet}\right)_{\mathrm{a}}(+\infty) \succ 0 \forall q \geqslant p$. Если число $q$ и степень импримитивности матрицы $A$ взаимно простые числа, то матрица $\left(F^{p \bullet}\right)(+\infty)=A^{q}$ будет неприводимой. Лемма доказана.

Вернемся к рассмотрению уравнения (1.1). Предполагается, что его предъядро является распределением абсолютно непрерывного типа. Функция $\varphi$ удовлетворяет следующему уравнению с предъядром $F^{q \bullet}$, где $q$ выбрано согласно требованиям леммы 4.2 :

$$
\varphi=g^{(q)}+\breve{F}^{q} \varphi
$$

где $g^{(q)}=g+\breve{F} g+\cdots+\breve{F}^{(q-1)} g$. Нужные нам свойства вектора $g$ легко переходят на вектор $g^{(q)}$. Если $m_{k}(d F)<+\infty$, то мера $d F^{q \bullet}$ также обладает конечным моментом порядка $k$. Из (4.19) и (4.10) следует свойство (4.16) для уравнения (4.20).

Применяя к уравнению (4.20) лемму 4.1, заключаем, что векторфункция $\varphi$ имеет структуру (4.17). В следующем параграфе будут найдены явные выражения для векторов $\mu$ и $\sigma=\int_{0}^{\infty} \omega(t) d t$.

\section{$\S 5$. Основная теорема}

5.1. Вычисление векторов $\mu$ и $\sigma$. Мы будем применять технику преобразования Лапласа. Обозначим (в отличие от §3) через $\hat{f}(s)=$ $\int_{0}^{\infty} f(t) e^{-s t} d t$ преобразование Лапласа функции $f$, при $s>0$ или $s \geqslant 0$.

Введем следующие обозначения, связанные с предъядром уравнения (1.1):

$$
A=F(+\infty), \quad F_{0}(t)=A-F(t) \geqslant 0 .
$$


Имеем

$$
\begin{gathered}
F_{0}(0)=A, \quad \tau_{k}\left(F_{0}\right)=\frac{1}{k+1} m_{k+1}(d F) \leqslant \infty, \quad k \geqslant 0 ; \\
\int_{0}^{\infty} e^{-s t} d F(t)=A-s \widehat{F}_{0}(s), \quad s \geqslant 0 .
\end{gathered}
$$

Пусть в уравнении (1.1) $g \in L_{1}^{n}$. Согласно формуле (4.17), имеем

$$
\begin{aligned}
& \widehat{\varphi}(s)=s^{-1} \mu+\widehat{\rho}(s)+\widehat{\psi}(s)=s^{-1} \mu+\widehat{\omega}(s), \\
& \widehat{\rho}(s)=o\left(s^{-1}\right), \quad \widehat{\psi}(s)=O(1), \quad \widehat{\omega}(s)=o\left(s^{-1}\right), \quad s \rightarrow 0+.
\end{aligned}
$$

Применяя к уравнению (1.1) преобразование Лапласа, с учетом (5.2) и (5.3) получаем

$$
\left[I-A+s \widehat{F}_{0}(s)\right]\left[s^{-1} \mu+\widehat{\omega}(s)\right]=\widehat{g}(s), \quad s>0 .
$$

Умножая обе части (5.4) на $s$ и устремляя $s$ к $0+$, приходим к следующему уравнению относительно $\mu:(I-A) \mu=0$, откуда с учетом (4.2) и $A \in P_{\mathrm{N}}($ см. (4.1)) получаем

$$
\mu=r \eta
$$

Постоянная $r$ подлежит определению.

Умножая (5.4) слева на вектор $\zeta$, с учетом (4.2) и (5.5) получаем

$$
r \zeta \widehat{F}_{0}(s) \eta+s \zeta F_{0}(s) \widehat{\omega}(s)=\zeta \widehat{g}(s) .
$$

Совершая предельный переход при $s \rightarrow 0$ приходим к равенству

$$
r=\varkappa^{-1} \zeta \int_{0}^{\infty} g(t) d t
$$

где $\varkappa=\zeta \nu_{1} \eta=\zeta m_{1}(d F) \eta \leqslant \infty$.

Если хотя бы одна компонента матрицы $m_{1}(d F)$ бесконечна, то $r=0$.

Займемся получением формулы для $\sigma=\int_{0}^{\infty} \omega(t) d t$ в случае конечного $m_{2}(d F)$. Из (5.4) с учетом (5.5) получаем

$$
(I-A) \sigma=\widehat{g}(0)-r m_{1}(d F) \eta .
$$

Равенство (5.7) обеспечивает выполнение условия

$$
\zeta\left[\widehat{g}(0)-r m_{1}(d F) \eta\right]=0
$$

разрешимости системы $(5.8)$ с необратимой матрицей $I-A$. Общее решение (5.8) имеет вид

$$
\sigma=\sigma_{0}+\lambda \eta
$$


где $\sigma_{0}$ - некоторое решение уравнения (5.8), а постоянная $\lambda$ подлежит определению.

Из (5.6) имеем

$$
s^{-1} r \zeta\left[\widehat{F}_{0}(s)-\widehat{F}_{0}(0)\right] \eta+\zeta \widehat{F}_{0}(s) \widehat{\omega}(s)=s^{-1} \zeta[\widehat{g}(s)-\widehat{g}(0)] .
$$

Совершая предельный переход при $s \rightarrow 0$, приходим к следующему выражению для постоянной $\lambda$ :

$$
\lambda=\frac{1}{2} \varkappa^{-2}\left[\zeta m_{2}(d F) \eta\right]\left[\zeta \int_{0}^{\infty} g(t) d t\right]-\varkappa^{-1} \zeta\left[m_{1}(d F) \sigma_{0}+\tau_{1}(g)\right] .
$$

Итак, вычислены важные величины $\mu$ и $\sigma$, связанные с представлением (4.17).

5.2. Основная теорема. Нами доказана следующая теорема для системы (1.1) с предъядром абсолютно непрерывного типа.

Теорема 5.1. Пусть предъядро уравнения многомерного восстановления (1.1) удовлетворяет условиям критичности (4.1) и является распределением абсолютно непрерывного типа. Тогда решение имеет структуру (4.17). Имеют место все утверждения леммы 4.1: векторьи $\eta$ и ऽ определяются в соответствии $c$ (4.2); вектор $\mu$ определяется согласно (5.5), коэффичиент $r$ определяется согласно (5.7). Вектор $\sigma=\int_{0}^{\infty} \omega(t) d t$ определяется по (5.9), где $\sigma_{0}$ является произвольным решением алгебраической системь (5.8), а коэффичиент $\lambda$ определяется из (5.11).

Пусть выполнены условия теоремы 5.1 и $m_{2}(d F)<\infty$. Тогда, согласно теореме 5.1, имеет место следующая асимптотическая формула типа формулы В. Смита (1.5):

$$
\int_{0}^{t} \varphi(x) d x=t \mu+\sigma+o(1), \quad t \rightarrow \infty .
$$

Теорема 5.1 включает в себе различные известные факты по скалярным УВ, их усиления и обобщения на многомерный случай. $\mathrm{K}$ числу таких фактов относится узловая теорема В. Смита (см. [7]-[9]) в частном случае распределения абсолютно непрерывного типа.

Рассмотрим теперь тот случай, когда мера $d F$ обладает конечными моментами до порядка $k \geqslant 3$ включительно. В теории восстановления задача построения третьего и последующих членов асимптотического разложения функции восстановления $H$ недостаточно полно изучена. На наш взгляд, утверждение теоремы 5.1 о конечности моментов функции $\omega$ до порядка $k-2$ является адекватной информацией о свойствах решения УВ в общем случае (если на структуру $F$ не накладываются дополнительные ограничения). В этом отношении представляет интерес вычисление моментов $\tau_{k}(\omega)$ функции $\omega$. При этом удобно воспользоваться 
следующей формулой, вытекающей из (1.1), (4.17) и (5.5):

$$
\tau_{k}(\omega)=\tau_{k}(g)-\frac{r}{k+1}\left[m_{k+1}(d F)\right] \eta+\sum_{p=0}^{k} C_{k}^{p} m_{p}(d F) \tau_{k-p}(\omega)
$$

\section{СПИСОК ЛИТЕРАТУРЫ}

1. Королюж В. С., Броди С. М., Турбин А. Ф. Полумарковские процессы и их применение. - Итоги науки и техники, сер. теория вероятн., матем. статист., теорет. кибернет., 1974, т. 11 , с. $47-97$.

2. Pyke R. Markov renewal processes: definitions and preliminary properties. - Ann. Math. Statist., 1961, v. 32, № 4, p. 1231-1242.

3. Jacod $J$. Théorème de renouvellement et classification pour les chaînes semimarkoviennes. - Ann. Inst. H. Poincaré, 1971, v. 7, № 2, p. 83-129; 1974, v. 10, p. 201-209.

4. Hunter J. J. On the renewal density matrix of a semi-Markov process. - Sankhyā, Ser. A, 1969, v. 31, № 3, p. 281-308.

5. Севастьянов Б.А., Чистяков В.П. Уравнения многомерного восстановления и моменты ветвящихся процессов. - Теория вероятн. и ее примен., 1971, т. 16, в. 2, c. 201-217.

6. Енгибарян Н.Б. Теоремы восстановления для системы интегральных уравнений. - Матем. сб., 1998, т. 189, № 12, с. 59-72.

7. Феллер В. Введение в теорию вероятностей и ее приложения. Т. 2. М.: Мир, 1984, $752 \mathrm{c}$.

8. Кокс Д., Смит В. Теория восстановления. М.: Советское радио, 1967, 299 с.

9. Севастьянов Б. А. Теория восстановления. - Итоги науки и техники, сер. теория вероятн. матем. статист., теорет. кибернет., 1974, с. 98-128.

10. Енгибарлн Н. Б. Уравнения восстановления на полуоси. - Изв. РАН, сер. матем., 1999 , т. 63 , № 1, c. 61-76.

11. Цалюк З.Б. Интегральные уравнения Вольтерра. - Итоги науки и техники, сер. матем. анализ, 1977, т. 15 , с. 131-198.

12. Ланкастер П. Теория матриц. М.: Наука, 1978, 280 с.

13. Крейн $М . \Gamma$. Интегральные уравнения на полупрямой с ядрами, зависящими от разности аргументов. - Успехи матем. наук, 1958, т. 13, № 5, с. 3-120.

14. Прёсдор $\oint$ 3. Некоторые классы сингулярных уравнений. М.: Мир, 1979, 493 с.

15. Григорян $Г$. А. Разрешимость одного класса интегральных уравнений ВинераХопфа. - Изв. НАН Армении, сер. матем., 1996, т. 31, № 2, с. 27-39. 